\section{AB0158 TAUROURSODEOXYCHOLIC ACID DECREASES THE EXPRESSION OF ERAD COMPONENTS AND THE ACCUMULATION OF SALIVARY MUCINS INDUCED BY PRO-INFLAMMATORY CYTOKINES}

N. Albornoz ${ }^{1}$, S. Aguilera ${ }^{2}$, M.-J. Barrera ${ }^{1}$, I. Castro ${ }^{1}$, P. Carvajal ${ }^{1}$,

S. González ${ }^{3}$, C. Molina $^{3}$, U. Urzúa ${ }^{1}$, D. Jara ${ }^{1}$, C. Leyton ${ }^{1}$, M.-J. González ${ }^{1}$.

${ }^{1}$ ICBM, Faculty of Medicine, University of Chile, ${ }^{2}$ Reumatología, Clínica Indisa;

${ }^{3}$ Patología Oral, Universidad Mayor, Santiago, Chile

Background: The salivary glands of Sjögren's syndrome patients show endoplasmic reticulum (ER) stress characterized by intracellular accumulation of secretory products such as $\mathrm{MUC1}^{1}$, dilated ER cisternae ${ }^{2}$ and high levels of pro-inflammatory cytokines. Previous results from our laboratory revealed an increase of the ATF6 $\alpha$ pathway of the UPR ${ }^{3}$ and activation of ER-associated protein degradation (ERAD) ${ }^{3}$. Increased expression of proteins involved in ERAD (SEL1L and EDEM1) has been reproduced in vitro in human submandibular gland (HSG) cells treated with TNF- $\alpha$ or IFN- $\gamma^{2}$. Tauroursodeoxycholic acid (TUDCA) is a chemical chaperone utilized for alleviate ER stress by enhancing the folding of proteins $^{3}$.

Objectives: The aim of this study was to evaluate if TUDCA decreases EDEM1, SEL1L, and MUC1 expression induced by pro-inflammatory cytokines in salivary gland epithelial cells.

Methods: HSG-cells were incubated with $10 \mathrm{ng} / \mathrm{mL}$ of IFN- $\gamma$ or TNF- $\alpha$ for $24 \mathrm{~h}$. Alternatively, cells were incubated with cytokines for $6 \mathrm{~h}$ and then co-incubated with TUDCA (150 and $250 \mu \mathrm{M})$ up to $24 \mathrm{~h}$. EDEM1, SEL1L and MUC1 protein and mRNA levels were determined by Western-blot and RT-qPCR, respectively. EDEM1 and SEL1L localization was determined by immunofluorescence.

Results: HSG cells stimulated with IFN- $\gamma$ or TNF- $\alpha$ showed a significant increase of EDEM1 and SEL1L protein and mRNA levels. Importantly, TUDCA co-incubation caused a significant decreased expression of both molecules. Treatment with both cytokines induced a cytoplasmic increase of staining intensity of EDEM1 and SEL1L, which was suppressed by TUDCA. HSG cells stimulated with cytokines showed a significant increase of MUC1 protein and mRNA levels, which was also suppressed by TUDCA.

Conclusions: Decreased expression of MUC1, SEL1L and EDEM1 in the presence of TUDCA suggests that this chemical chaperone promotes folding of proteins in the ER, by decreasing ERAD activity and ER stress induced by pro-inflammatory cytokines in HSG cells. These results enable us to propose that TUDCA might alleviate the ER stress of salivary glands from Sjögren's syndrome patients.

References:

[1] Oral Dis. 2015;21(6):730-8

[2] Arthritis Rheum. 2003 Sep;48(9):2573-84.

[3] J Autoimmun. 2016:75:68-81.

[4] Prion. 2014;8(2). pii: 28938.

Acknowledgements: Supported by Fondecyt-Chile [\#1160015] (MJG, SA, CM, SG, IC).

Disclosure of Interest: None declared

DOI: 10.1136/annrheumdis-2017-eular.2003

\section{AB0159 AUTOIMMUNE REACTIVITY TO MALONDIALDEHYDE ADDUCTS IN SYSTEMIC LUPUS ERYTHEMATOSUS}

U. Hardt ${ }^{1}$, A. Larsson ${ }^{2}$, I. Gunnarsson ${ }^{1}$, G.J. Silverman ${ }^{3}$, E. Svenungsson ${ }^{1}$, C. Grönwall ${ }^{1}{ }^{1}$ Dept. of Medicine, Rheumatology Unit, Karolinska Institute and Karolinska University Hospital, Stockholm, Sweden; ${ }^{2}$ Dept. of Medical Sciences, Clinical Chemistry, Uppsala University, Uppsala, Sweden; ${ }^{3}$ Dept. of Medicine, NYU School of Medicine, New York, NY, United States

Background: Systemic Lupus Erythematosus (SLE) is an autoimmune disease characterized by recurrent disease activity flares, multiple organ involvement, and often with a presentation of nephritis. Malondialdehyde (MDA) post-translational modification of proteins occurs upon inflammation and oxidative stress. Natural IgM anti-MDA autoreactivity is present from birth and may be beneficial. However, both IgM and IgG anti-MDA can also be increased in autoimmune disease. Yet, the role for potentially pro-inflammatory autoreactive IgG anti-MDA in SLE remains elusive.

Objectives: Here, we study the association between serum IgG anti-MDA and clinical features of SLE.

Methods: This cross-sectional study included 398 SLE patients at the Karolinska University Hospital fulfilling at least four of the 1982 ACR criteria. Data was compared to the previously reported combined US east coast cohorts $(1,2)$. Disease activity was assessed by SLEDAl-2K. Total IgG and ANA IgGs were assessed in the clinical laboratory and STNFR by ELISA. IgG anti-MDA was measured by a quantitative ELISA using modified BSA. As a comparison, we measured IgG anti-phosphorylcholine (PC), another oxidation-associated natural IgG. Cutoff for positivity was based on highest control quartile. Specific IgGs were normalized for total lgG levels in the analysis. Means were compared with Mann-Whitney test, correlations with Spearman's analysis, and meta-analysis used Mantel-Haenszel fixed effect model.

Results: Serum IgG anti-MDA significantly correlated with SLE-associated autoIgG (e.g. anti-dsDNA, $n=398 \quad R=0.42 p<0.0001$ ). IgG anti-MDA correlated with higher disease activity by SLEDAI in two independent cohorts (Sweden KS, $n=397 R=0.33 \quad p<0.0001$; US east coast, $n=219 R=0.34 p<0.0001$ ) and was confirmed in meta-analysis of dichotomized data showing an Odds Ratio of 3.9 ( $\mathrm{Cl}$ 2.6-5.8 $p<0.0001)$ for $\lg G$ anti-MDA positivity in patients with active disease (SLEDAI $\geq 6$ ). Association of anti-MDA with disease activity was supported by an inverse correlation of IgG anti-MDA normalized for total IgG with complement (C2, $n=304 R=-0.24 p<0.0001, C 3$ and $C 4, n=385 R=-0.24 p<0.0001$ ). Furthermore, we observed elevated IgG anti-MDA/total IgG reactivity in SLE patients with current or history of nephritis compared to no history of nephritis $(n=3894.5+/-4.0$ vs $3.4+/-3.3 p<0.0001$ ) and inverse weak correlation of IgG anti-MDA/total IgG with markers of kidney function (serum cystatin $C, n=283 R=0.20 p=0.0008$, urine albumin, $n=373 \quad R=0.23 \quad p<0.0001$ ). Anti-MDA IgG/total IgG also directly correlated with serum soluble TNF receptors (sTNFR1, $n=286 \mathrm{R}=0.21 \mathrm{p}=0.0003$, sTNFR2, $\mathrm{n}=287 \mathrm{R}=0.35, \mathrm{p}<0.0001)$. IgG anti-PC either did not correlate or inversely correlated with disease measurements, consistent with its previously reported more protective properties.

Conclusions: IgG to MDA-modifications correlates with other autoreactivities, disease activity and nephritis in SLE, and should be further evaluated for its potential prognostic utility. Yet, it remains unclear, if IgG anti-MDA directly contributes to pathogenesis in SLE.

References:

[1] Clin Immunol. 2012 Mar;142(3):390-8.

[2] Clin Immunol. 2014 Jul;153(1):1-7.

Disclosure of Interest: None declared

DOI: 10.1136/annrheumdis-2017-eular.5822

\section{AB0160 INTERFERON-Г-INDUCIBLE KYNURENINES INFLAMMATION PATHWAY: THE MISSING LINK BETWEEN DISEASE ACTIVITY AND SYMPTOMS IN SJÖGREN'S SYNDROME}

V. Valim ${ }^{1}$, W.M. Sardemberg ${ }^{1}$, J.G. Brun ${ }^{2}$, E. Zandonade ${ }^{3}$, G.M. Balarini ${ }^{1}$, L.V. Tanure ${ }^{4}$, G.V. Ferreira ${ }^{4}$, É.V. Serrano ${ }^{1}$, J.F.V. Tonini ${ }^{1}$, K.A. Brokstad ${ }^{5}$, P.M. Ueland ${ }^{6}$, R. Jonsson ${ }^{5}$, P.M. Mydel ${ }^{5} .{ }^{1}$ Medicine, Federal University of Espírito Santo, Vitória, Brazil; ${ }^{2}$ Clinical Science Department, University of Bergen, Bergen, Norway; ${ }^{3}$ Statistic Department, Federal University of Espírito Santo; ${ }^{4}$ Locomotor System Department, Federal University of Minas Gerais, Vitória, Brazil; ${ }^{5}$ Broegelmann Research Laboratory, University of Bergen, Bergen, Norway; ${ }^{6}$ Bevital Laboratory A/S, University of Bergen, Vitória, Brazil

Background: Tryptophan (TRYP) can be converted to kynurenine (KYN) by indoleamine 2,3-dioxygenase (IDO) drived by interferon- $\gamma$. Recent studies have suggested that the KYN pathway reflects an important interface between the immune and nervous system modulation.

Objectives: The aim was to study KYN pathway and their correlation to clinical and immunological parameters in primary Sjögren's syndrome (pSS).

Methods: We included 97 pSS (AECG) and 63 healthy controls matched to age, sex, ethnicity, and body mass index (BMI). KYN metabolites and TRYP were analysed by liquid chromatography mass spectrometry.

Results: Patients aged $50 \pm 11$ years showed anti-SSA-Ro of $63 \%$, anti-SSB-La $31 \%$, anti-nuclear antibody $81 \%$, rheumatoid factor $24 \%$, and systemic manifestations $67 \%$. Most (68\%) showed low disease activity measured by Eular Sjögren's Syndrome Disease Activity Index (ESSDAI), 22\% moderate and 10\% high ESSDAl. The kynurenine:tryptophan ratio (KTR) was $(0.031 \pm 0.014$ vs. $0.024 \pm 0.007, p=0.001)$, $K Y N(1.890 \pm 0.580$ vs. $1.652 \pm 0.426, p=0.005)$, quinolinic acid (QA) (477.82 2251.55 vs. $382.05 \pm 128.06, p=0.018)$, hydroxikynurenine (3HK) $(53.45 \pm 52.05$ vs. $39.15 \pm 9.67, p=0.056)$, anthranilic acid (AA) $(19.86 \pm 6.26$ vs. $16.78 \pm 4.71, p=0.001)$ were higher while xanthurenic acid $(X A)(11.52 \pm 7.88$ vs.13.00 $\pm 5.68, p=0.019)$, and TRYP $(64.90 \pm 13.43$ vs. $71.02 \pm 8.88, p=0.012)$ were lower in pSS compared to controls. Higher KTR was associated with disease duration $(r=0.211, p=0.042)$, CRP $(r=0.254, p=0.029)$, lower hemoglobin $(r=-$ $0.219, p=0.34)$, creatinine $(r=0.588, p=0.000)$, hypergammaglobulinemia $(r=0.254$, $\mathrm{p}=0.014)$, hyper IgG $(\mathrm{r}=0.354, \mathrm{p}=0.004)$, lower $\mathrm{C} 3(\mathrm{r}=0.262, \mathrm{p}=0.011)$ and $\mathrm{C} 4$ $(r=-0.294, p=0.004)$. Higher KTR was observed in those with Biological ESSDAI domain involvement $(0.033 \pm 0.016$ vs. $0.029 \pm 0.013, p=0.003)$, glandular manifestation $(0.037 \pm 0.014$ vs. $0.029 \pm 0.013, p=0.007)$, in the other hand lower KTR in those with presence of musculoskeletal pain $(0.029 \pm 0.011$ vs. $0.032 \pm 0.015$, $p=0.003)$. ESSDAl showed a tendency to correlate with KTR $(r=0.177, p=0.091)$ and ESSPRI inversely correlated with $A A(r=-0.233, p=0.071)$. Either patient with pain showed lower $A A(20.66 \pm 6.66$ vs. $17.22 \pm 5.07, p=0.021)$.

Conclusions: TRYP is decreased and KYN metabolites pathway is increased in pSS. IDO activity expressed like KTR was positively correlated with disease activity and glandular manifestations but negatively with pain. A better understanding of the KYN pathway can clear the dissociation of symptoms and disease activity in pSS.

\section{References:}

[1] Maria NI, van Helden-Meeuwsen CG, Brkic Z, et al. Association of Increased Treg Cell LevelsWith Elevated Indoleamine 2,3-Dioxygenase Activity and an Imbalanced KynureninePathway in Interferon-Positive Primary Sjögren's Syndrome. Arthritis Rheumatol.2016 Jul:68(7):1688-99.

[2] Midttun $\varnothing$, Hustad S, Ueland PM. Quantitative profiling of biomarkers relatedto B-vitamin status, tryptophan metabolism and inflammation in human plasma byliquid chromatography/tandem mass spectrometry. Rapid Commun Mass Spectrom. 2009 May;23(9):1371-9.

Disclosure of Interest: None declared 
DOI: 10.1136/annrheumdis-2017-eular.6015

\section{AB0161 ANTIPHOSPHOLIPID ANTIBODIES DIFFERENTIALLY REGULATE THE EXPRESSION \& ACTIVITY OF THE LYSOSOMAL PROTEASES WITH EFFECTS UPON MONOCYTE AUTOPHAGY}

V.M. Ripoll-Nunez, A. Khawaja, I.P. Giles, A. Rahman. Division of Medicine, University College London, London, United Kingdom

Background: Antiphosholipid antibodies (aPLs) are known to activate monocytes in the pathogenesis of antiphospholipid syndrome (APS), although the precise mechanisms by which this activation occurs are not fully understood. We have recently identified several novel protein targets using a comprehensive proteomic analysis of human monocytes treated with lgG from patients with APS. Amongst these novel targets lysosomal proteases, including cathepsin B and cathepsin $\mathrm{D}$ were identified. These proteases are important in protein degradation, clearance of autolysosomes, apoptosis and autophagy. Dysregulation of these homeostatic cellular functions may be important in the exposure of autoantigens and pathogenesis of the APS. Therefore, we have now studied the effects of APS IgG upon the expression/activity of different cathepsins and their effects upon autophagy.

Objectives: Determine the effect of pathogenic aPL antibodies on monocyte autophagy and its association with the regulation of lysosomal activity.

Methods: Healthy monocytes were treated with $200 \mu \mathrm{g} / \mathrm{ml}$ of $\mathrm{lgG}$ purified from $(n=9)$ patients with APS or $(n=9)$ healthy control $(H C)$ IgG for 6 h. The expression of cathepsin B and cathepsin D were measured by western blotting. Activity assays for lysosomal proteases cathepsin D, cathepsin $B$ and cathepsin $L$ were performed using fluorescence based assays (RayBio ${ }^{\circledR}$ ). Intracellular proteolytic activity of monocytes was determined using DQ-BSA (Molecular probes) and flow cytometry analysis. Autophagy was induced by treating monocytes with $50 \mu \mathrm{g} / \mathrm{ml}$ GM-CSF for $14 \mathrm{~h}$.

Results: Consistent with our previous label free quantification mass spectrometry proteomic analysis, western blot analysis confirmed that levels of cathepsin B and cathepsin D were decreased in monocytes treated with APS IgG compared to $\mathrm{HC}$ IgG. Similarly, enzymatic assays revealed that cathepsin B and cathepsin $D$ activities were significantly reduced in monocytes treated with IgG from patients with APS compared to $\mathrm{HC}(\mathrm{p}=0.0188,0.0323)$. In contrast, levels of enzymatic activity of cathepsin $L$ were increased in monocytes treated with APS $\lg$ compared to $\mathrm{HC} \lg G(p=0.0106)$. To determine the effect of APS IgG on autophagy, we exposed healthy monocytes to $\lg G$ and induced autophagy by treating them with GM-CSF for $14 \mathrm{~h}$. Subsequently we tested the intracellular proteolytic activity with DQ-BSA. Stimulation of monocytes with APS IgG reduced the lysosomal activity of GM-CSF-treated monocytes whereas HC IgG had no effect, indicating that APS IgG disrupts lysosomal degradation during monocyte autophagy.

Conclusions: We found that IgG from patients with APS regulate the expression and activity of lysosomal proteases cathepsins $B / D$ and cathepsin $L$ in opposite directions. Activity of cathepsin $B$ and $D$ was down-regulated by exposure to $\mathrm{IgG}$ from patients with APS whereas cathepsin $\mathrm{L}$ was up-regulated. Furthermore, we found APS IgG disrupts lysosomal degradation during monocyte autophagy. Additional experiments are now underway to increase our understanding of how modulation of cathepsin activity and autophagy may be important in the pathogenesis of APS and provide new therapeutic targets.

Disclosure of Interest: None declared

DOI: 10.1136/annrheumdis-2017-eular.3417

\section{AB0162 SERUM LEVEL OF PROINFLAMMATORY CYTOKINES IS NEGATIVELY ASSOCIATED WITH FATIGUE IN PRIMARY SJÖGREN'S SYNDROME}

D. Gardiner ${ }^{1}$, W.D. Reid ${ }^{2}$, J.R. Tarn ${ }^{1}$, D. Lendrem ${ }^{1}$, N. Howard-Tripp ${ }^{1}$,

S. Bowman ${ }^{3}$, B. Griffiths ${ }^{4}$, S. Rushton ${ }^{2}$, W. .F. Ng ${ }^{1}$ on behalf of UKPSSR

${ }^{1}$ Institute of Cellular Medicine \& NIHR Newcastle Biomedical Research Centre;

${ }^{2}$ School of Biology, Newcastle University, Newcastle upon Tyne; ${ }^{3}$ Rheumatology, University Hospital Birmingham, Birmingham; ${ }^{4}$ Rheumatology, Freeman Hospital, Newcastle upon Tyne, United Kingdom

Background: We have previously described a model of fatigue in patients with primary Sjogren's syndrome (PSS) based on the levels of serum cytokines, pain and depression scores. Importantly, removal of cytokines from this model substantially reduced the accuracy suggesting that cytokines may have a key role in the biological basis of fatigue [1]. However, interpreting the model is complicated by the complexity of the immune system and the likely multiple interactions between numerous cytokines and other variables [2]. Structural equation modelling (SEM) is a statistical technique that allows for analysis of one or multiple independent variables with one or multiple dependent variables. SEM consists of two components - the structural model, which represents the relationships between the theoretical variables, and the measurement model, which are the relationships between the latent variables and their measures [3].

Objectives: To use SEM to test our hypothesis that the balance between pro-inflammatory and anti-inflammatory cytokines play an important role in determining severity of fatigue in patients with PSS.
Methods: We used Canonical Correpondence Analysis (CCA) to investigate the variation in cytokine expression across our spectrum of fatigue patietns to explore interactions and dependencies between cytokines. We then built a conceptual model based on the literature representing the likely relationships between fatigue and various proinflammatory and anti-inflammatory cytokines and other soluble molecules in the serum. This conceptual model was then challenged using serum data and fatigue scores of 161 PSS patients from the UK primary Sjogren's syndrome registry. Model fit was assessed using the Confirmatory Factor Index, the Root Mean Square Error of Association and the Standardised Root Mean Square Residual. We also analysed changes in fatigue scores over a period between 1-4 years.

Results: CCA revealed the first axis of ordination (CCA1) broadly correlates with fatigue, consists of many pro-inflammatory cytokines including TNF $\alpha, L T \alpha$ and IFN $\gamma$, IL17, which were negatively correlated with fatigue while IL-6 and MCP1, which were positively associated with increased fatigue severity. The second axis (CCA2) reflects a trend in cytokines which appear to relate to patients' age. Fatigue scores were largely stable over time and therefore data were not included in the SEM analysis. The main pro-inflammatory SEM model showed fatigue was negatively associated with pro-inflammatory cytokine activity $(p=0.019) ; \mathrm{IL}-10$ drove IP-10 $(p=0.000)$; and IL-10 was driven by IL-6 $(p=0.006)$ (Fig. 1)

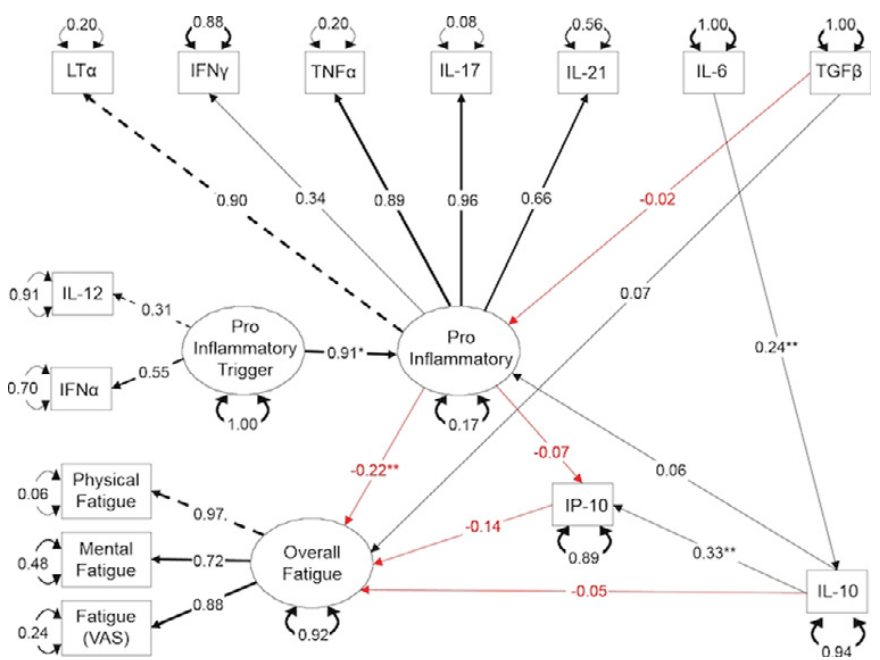

Conclusions: Chronic fatigue in PSS is negatively associated with many pro-inflammatory cytokines. We hypothesize that it reflects adaptive biological processes, which occurs after chronic exposure to inflammation in conditions such as PSS.

References:

[1] Howard-Tripp N, et al. RMD Open. 2016;2:e000282.

[2] Genser B, et al. BMC immunology. 2007;8:1.

[3] Beran TN \& Violato C. BMC Research Notes. 2010;3:267.

Acknowledgements: All UKPSSR participants.

Disclosure of Interest: D. Gardiner: None declared, W. Reid: None declared, J. Tarn: None declared, D. Lendrem: None declared, N. Howard-Tripp: None declared, S. Bowman Consultant for: Cellgene, Glenmark, GSK, Eli Lilly, Novartis, Roche, Takeda, UCB, B. Griffiths: None declared, S. Rushton: None declared, W.-F. Ng Consultant for: Pfizer, UCB, Medlmmune, Takeda and Sanofi

DOI: 10.1136/annrheumdis-2017-eular.5811

\section{AB0163 MESENCHYMAL STEM CELLS INDUCE CD1C+ TOLEROGENIC DENDRITIC CELLS IN HUMAN SYSTEMIC LUPUS ERYTHEMATOSUS VIA UP-REGULATING FLT-3 LIGAND}

X. Yuan, D. Wang, Z. Zhang, Q. Wang, W. Li, X. Tang, W. Chen, G. Yao, L. Sun. Department of Rheumatology and Immunology, The Affiliated Drum Tower hospital of Nanjing University Medical School, Nanjing, China

Background: $\mathrm{CD}_{1 \mathrm{c}^{+}}$tolerogenic dendritic cells (DCs) play important roles in the induction of peripheral tolerance and control of adaptive immune response. Umbilical cord (UC)-derived mesenchymal stem cells (MSCs) exhibit immunoregulation effects in systemic lupus erythematosus (SLE). However, the underlying immunosuppression mechanism of MSCs via tolerogenic DCs in SLE remains largely unknown.

Objectives: The aim of this study was to examine tolerogenic DCs levels in SLE patients, and to further investigate the mechanism of MSCs in the regulation of tolerogenic DCs.

Methods: Tolerogenic DCs were isolated as Lin (CD3/19/56/14)- HLA $\mathrm{DR}^{+} \mathrm{CD} 11 \mathrm{c}^{+} \mathrm{CD} 1 \mathrm{c}^{+}$from peripheral blood mononuclear cells (PBMCs). Levels of tolerogenic DCs were determined by flow cytometry, and serum concentration of FIt-3 ligand (FLT3L) were determined by ELISA from 17 healthy controls and 25 SLE patients. Eight SLE patients were given UC MSCs infusions. We compared the levels of tolerogenic DCs and serum FLT3L before and 24 hours after UC 